\title{
Evolutionary diversification of Japanese Stomaphis aphids (Aphididae, Lachninae) in relation to their host plant use and ant association
}

\author{
Tetsuya Yamamoto $^{1}$ (D) Mitsuru Hattori $^{2} \cdot$ Yoshiyuki Matsumoto $^{3} \cdot$ Shouhei Ueda $^{4} \cdot$ Takao Itino $^{5}$
}

Received: 7 November 2019 / Revised: 25 February 2020 / Accepted: 2 March 2020 / Published online: 19 March 2020

(C) The Author(s) 2020

\begin{abstract}
Phytophagous insects are among the most diverse of the earth's organisms, and their diversification patterns and the driving forces behind these have attracted considerable research interest. Host shifting to closely related plant species is thought to play an important role in phytophagous insect diversification, but the extent to which other interactions such as mutualistic associations affect diversification is not yet known. In this study, we reconstructed the molecular phylogeny of Japanese Stomaphis aphids and determined whether host shifting or mutualistic association with different ant species could explain diversification in this aphid genus. We analyzed 12 species of Stomaphis and grouped them into ten well-supported DNA lineages. Species in each lineage used a single or a few host plant species, but were mutualistically associated with many ant species of the genus Lasius. This result suggests that Stomaphis evolutionarily diversified primarily through host plant shifts. Interestingly, the reconstructed phylogeny suggests that Stomaphis host shifts occasionally occurred between very distantly related host plant taxa (spanning up to five plant orders). The dependence of Stomaphis on long-lasting Lasius ant colonies situated in temperate deciduous forests where Lasius is the dominant ant genus may have led the aphids to shift to distantly related but spatially adjacent host tree species.
\end{abstract}

Keywords Host shift · Phylogenetic reconstruction $\cdot$ Phytophagous insect $\cdot$ Species specificity

Communicated by: Oliver Hawlitschek

Electronic supplementary material The online version of this article (https://doi.org/10.1007/s00114-020-1671-4) contains supplementary material, which is available to authorized users.

Tetsuya Yamamoto

8mate2@gmail.com

1 Interdisciplinary Graduate School of Science and Technology, Shinshu University, Nagano, Japan

2 Graduate School of Fisheries and Environmental Sciences, Nagasaki University, Nagasaki, Japan

3 Shibaura Institute of Technology Kashiwa Junior and Senior High School, Chiba, Japan

4 Graduate School of Life and Environmental Science, Osaka Prefecture University, Osaka, Japan

5 Department of Biology, Faculty of Science, Shinshu University, Nagano, Japan

\section{Introduction}

One of the goals of evolutionary biology is to understand the factors and mechanisms that have led to species diversity on the earth. Insects are among the most diverse taxa described thus far, and phytophagous insects account for more than $40 \%$ of all described insects (Grimaldi and Engel 2005). Therefore, the patterns and driving forces of their diversification have been studied extensively (Agosta 2006; Futuyma and Agrawal 2009; Depa et al. 2017).

Phytophagous insects are hypothesized to have diversified by ecological speciation through adaptation to different, often closely related host plant species, followed by the interruption of gene flow owing to disruptive selection. Many studies have provided evidence supporting this hypothesis, both by reconstructing phylogenies of phytophagous insects to examine the evolutionary diversification of their host plant use (Funk et al. 1995; Peccoud et al. 2009) and by comparing host preference and performance (e.g., growth rate) among host lineages (Nosil et al. 2002; Matsubayashi et al. 2011, 2013; Fujiyama et al. 2013). For example, Peccoud et al. (2009) 
assessed clonal lineages of the pea aphid, Acyrthosiphon pisum, and found eight sympatric host lineages, each of which was specialized to a different host plant species.

Interspecific interactions other than those with host plants can also drive the diversification of phytophagous insects (Pierce et al. 2002). For example, Depa et al. (2017) reported that two sister aphid species diverged because of their association with different ant species. Phytophagous insect diversification through interactions with organisms other than host plants is still poorly understood; however, the relative importance of host plants and other organisms in structuring insect evolutionary diversification needs to be elucidated.

Phytophagous aphids are highly specific to host plants; $99 \%$ of aphid species use a single specific plant species or a few closely related plant species (Blackman and Eastop 1994; Dixon 1998). Furthermore, even aphid species that appear to be "generalists" may be genetically differentiated into different host lineages, as in the case of A. pisum mentioned above (Peccoud et al. 2009). Therefore, aphid diversity has generally been attributed to adaptive diversification to different closely related host plant species (Dixon 1998; Drès and Mallet 2002).

Aphids also establish mutualistic relationships with ants by providing them with honeydew in return for protection from natural enemies and hygienic services (Hölldobler and Wilson 1990; Stadler and Dixon 2005), and some aphid traits have evolved owing to the selection pressure from attending ants: for example, an anal plate shaped to hold honeydew (Heie 1987; Kanturski et al. 2017), a long proboscis and stylet (Shingleton et al. 2005), or a particular cuticular hydrocarbon profile (Lang and Menzel 2011). In addition, some aphid traits are plastically induced by the presence of attending ants: for example, production of more and higherquality honeydew (Fischer and Shingleton 2001) or a smaller flight apparatus (Yao 2012). These adaptations and the fact that different ant species offer different degrees of protection to the aphids with which they associate (Novgorodova 2005) suggest that adaptive diversification to particular ant species might occur.

The genus Stomaphis is a group of large aphids having about 4-7 mm of body length; they also have a long proboscis and stylet, which, in adult females, may be up to twice the body length (Brożek et al. 2015). Stomaphis aphids use this long mouthpart to suck phloem sap from tree trunks. Thirtythree species and four subspecies of Stomaphis have been described worldwide, and most have been described as specific to a single plant species or genus (Blackman and Eastop 2019). However, the morphological classification of Stomaphis may not accurately reflect the phylogenetic relationships within the genus Stomaphis; for example, Depa et al. (2012) reported discrepancies between the molecular phylogeny of some Stomaphis species and their morphological classification.
Globally, host plants used by aphids of the genus Stomaphis belong to 13 families in seven orders, although most aphid species usually specialize to a single or few closely related plant species (Blackman and Eastop 2019). This huge taxonomic breadth of host plant usage suggests that Stomaphis aphids may have diversified through host plant shifts, occasionally between very different taxa. Indeed, European Stomaphis species comprise two sister mtDNA lineages, each of which uses a different and distantly related host plant species (Sapindales and Malpighiales; Depa and Mróz 2013), suggesting that diversification occurred by a host plant shift.

Importantly, Stomaphis aphids interact with not only plants but also ants, with which they have a mutualistic relationship. The long mouthparts of Stomaphis aphids restrict their mobility, rendering it difficult for them to escape from their natural enemies. Because Stomaphis aphids strongly depend on attending ants for protection from predators and receiving hygienic services, they cannot survive without ants (Lorenz and Scheurer 1998). In at least one case, aphid diversification resulted from such aphid-ant interaction; Depa et al. (2017) reported that the sister species Stomaphis quercus and S. wojciechowskii share the same host plant species, but each maintains a mutualistic relationship with a different ant species. Each aphid species has evolved morphological and ecological characteristics suitable for interaction with its own partner ant species. For example, a population of $S$. quercus associated with ant species that is a partner of $S$. wojciechowskii acquires morphological characters same as those of $S$. wojciechowskii. Such ant-related diversification in Stomaphis aphids suggests that aphid-ant mutualism can lead to the diversification of these phytophagous insects. However, this phenomenon has been recognized in only these two Stomaphis species, and whether this mode of diversification occurs more generally in this genus is not yet known.

This study aimed to elucidate the influence of interactions with host plants and associated ants on phylogenetic diversification in the genus Stomaphis. First, we reconstructed the phylogeny of Stomaphis species in Japan from mitochondrial and nuclear DNA sequences and then investigated the relationships between the phylogenetic lineages and host plant utilization and ant association. The results suggested that diversification in Japanese Stomaphis aphids occurred through interactions with host plants rather than with associated ants.

\section{Methods}

\section{Field sampling}

We discovered 160 Stomaphis aphid colonies at 34 sites in Japan by searching for known host plants and/or by following Lasius ant trails (Table S1). We considered all aphids on a 
single host plant to belong to a single colony. The aphids and associated ants were collected from each host plant and stored in $99.5 \%$ and $70 \%$ ethanol, respectively, at $4{ }^{\circ} \mathrm{C}$ before DNA extraction and morphological identification.

\section{Interspecific relationships}

To clarify the correspondence between aphid phylogeny and aphid interspecific interactions, we identified all host plants and attending ant species. Host plants were identified on the basis of leaf and stem morphology, and associated ant species were identified on the basis of their mitochondrial COI sequences, because it is often difficult to identify Lasius ants at the species level on the basis of morphology alone. Protocols and primers for mitochondrial COI sequence analyses of Lasius ants were referred from Maruyama et al. (2008).

\section{DNA extraction and sequencing of aphids}

Total genomic DNA was extracted from a single aphid by using a DNeasy Blood \& Tissue Kit (Qiagen) following manufacturer's instructions. We targeted two molecular markersmitochondrial cytochrome oxidase c subunit II (COII) and exon of nuclear elongation factor $1 \alpha(E F-1 \alpha)$. The $C O I I$ gene was amplified using polymerase chain reaction (PCR) analysis and Takara Tks Gflex DNA polymerase (Takara Bio, Shiga, Japan) by using the PCR primer set mt2993+ $\left(5^{\prime}-\right.$ CATTCATATTCAGAATTACC-3') and Eva-R (5'-GAGA CCATTACTTGCTTTCAGTCATCT-3'; Brower and Jeansonne 2004; Stern 1994). The EF-1 $\alpha$ gene was amplified using PCR with Takara Ex Taq DNA polymerase (Takara Bio, Shiga, Japan) by using the PCR primer set efs 175 (5'-GGAA ATGGGAAAAGGCTCCTTCAAGTAYGCYTGGG-3') and efa1082 (5'-ATGTGAGCAGTGTGGCAATCCAA-3'; Normark 1999). The PCR temperature profile was 30 cycles at $98^{\circ} \mathrm{C}$ for $10 \mathrm{~s}, 50{ }^{\circ} \mathrm{C}$ for $10 \mathrm{~s}$, and $72{ }^{\circ} \mathrm{C}$ for $60 \mathrm{~s}$ for $\mathrm{COII}$ and 30 cycles at $98{ }^{\circ} \mathrm{C}$ for $10 \mathrm{~s}, 42^{\circ} \mathrm{C}$ for $30 \mathrm{~s}$, and $72{ }^{\circ} \mathrm{C}$ for $60 \mathrm{~s}$ for $E F-1 \alpha$. After amplification, the PCR product was purified using ExoSap-IT reagent (USB; Cleveland, OH, USA). Cycle sequencing reactions for both strands were performed using a BigDye Terminator version 1.1 Cycle Sequencing Kit (ABI, Weiterstadt, Germany) on an ABI 3130 Genetic Analyzer.

\section{Phylogenetic analyses}

The mitochondrial COII and $E F-1 \alpha$ sequences of $589 \mathrm{bp}$ $(C O I I)$ and 723 bp $(E F-1 \alpha)$ were edited and aligned using the SeqScape v. 2.5 software (ABI; Weiterstadt, Germany). We selected the best-fit substitution model by using Bayesian information criterion 4 (BIC4) in a Kakusan4 software package (Tanabe 2007): for COII, we used $\mathrm{J} 2+\mathrm{G}$ for the first and second codon positions and $\mathrm{J} 1+\mathrm{G}$ for the third codon position; for $E F-1 \alpha$, we used HKY $85+\mathrm{G}$ for the first and third codon positions and JC69 $+\mathrm{H}$ for the second codon position. We performed a maximum likelihood analysis by using TREEFINDER version October 2008 software (Jobb et al. 2004) and the substitution models selected above. Clade support was assessed using 1000 bootstrap replications by using TREEFINDER. The mitochondrial COII genetic distance was calculated using Kimura 2-Parameter (K2P) model by using Mega7 (Kumar et al. 2016). Next, we identified host plants and attending ants associated with each reconstructed phylogenetic lineage. The mitochondrial (COII) haplotype network estimated using the median-joining network (MJ) method was constructed using PopART ver. 1.7 (Leigh and Bryant 2015). To facilitate the understanding of the relationship between Stomaphis aphids and host plants, we reconstructed the character of host plant use on a haplotype network.

\section{Morphological identification}

To identify aphid species on the basis of morphology, we collected apterous viviparous or oviparous adult females from one or few Stomaphis colonies belonging to each phylogenetic lineage or (if members of the lineage used more than one host plant species) from each host plant species. Each sample was immersed in $10 \% \mathrm{KOH}$ and encapsulated in a Canada balsam by using the method of Kozarzhevskaya (1986). We identified morphological species by referencing to taxonomic and biological traits (Inouye 1938; Takahashi 1960; Sorin $1965,1979,1995)$ and by measuring each part of the aphid's body under an optical microscope following the key to Japanese Stomaphis species (Sorin 2012). All slide samples are now in Matsumoto's collection.

\section{Results}

\section{Morphological identification}

For measuring morphological features of Stomaphis aphids, 12 morphological species (S. abieticola, S. aceris, S. aphananthae, S. fagi, S. hirukawai, S. japonica, S. malloti, S. matsumotoi, S. pterocaryae, S. takahashii, $S$. ulmicola, and S. yanonis), two subspecies (S. pini takaoensis and S. yanonis aesculi), and three undescribed species (Stomaphis spp. 1-3) were identified (Table S1). Of the 15 species and three subspecies described in Japan, three species (S. alni, S. carpini, and S. pini) and one subspecies (S. asiphon sakuratanii) were not available. 


\section{Phylogeny of Stomaphis aphids}

The combined sequence matrix used for phylogenetic reconstruction was 1312 bp long. The collected Japanese Stomaphis specimens were grouped into ten major DNA lineages (A to J) with a COII genetic distance by K2P model greater than 0.03 (Fig. 1). Each DNA lineage was supported by a ML bootstrap value with a probability of more than $70 \%$ and included one or more previously described species: lineage A (S. aphananthae, S. malloti, and S. yanonis); lineage B (samples for morphological identification could not be obtained); lineage C ( $S$. aceris and $S$. takahashii); lineage D (S. pterocaryae, S. yanonis aesculi, and Stomaphis sp. 1); lineage E (S. matsumotoi); lineage F ( $S$. fagi); lineage G (S. japonica and Stomaphis sp. 2); lineage H (S. abieticola, S. japonica, S. pini takaoensis, and Stomaphis sp. 3); lineage I (S. hirukawai); and lineage J (S. ulmicola). Lineages D and H were subdivided into three and five sublineages, respectively, according to host plant usage; each sublineage utilizes a different host plant species (Fig. 1). Because lineage B consisted of a single sample, we refrain from discussing its relationship to plants and ants.

\section{Host plant use}

Each of the five Stomaphis aphid lineages (C, E, F, I, and J) used a single plant species as a host, whereas each of the other four lineages (A, D, G, and $\mathrm{H}$ ) used three or more host plant species (Fig. 2, Table S2). Each sublineage in lineages D and H (D-I, D-II, D-III, H-I, H-II, H-III, H-IV, and H-V), except HI, used a single host plant species. Host plant species mostly did not overlap among the lineages or sublineages, except between sublineages H-I and H-II. In all, the Stomaphis aphids used 22 host plant species belonging to 15 genera, ten families, and five orders, and the evolutionary host plant shift associated with aphid speciation was overwhelmingly wide (between orders; Table S2). To our knowledge, this is the first study to document Betula ermanii, Picea jezoensis var. hondoensis, Quercus crispula, and Q. dentata as host plant species of Stomaphis aphids in Japan (Inouye 1938; Takahashi 1960; Sorin 1965, 1979, 1995, 2012).

\section{Haplotype analysis}

From the 160 COII sequences of Stomaphis aphids, a total of 38 haplotypes were identified (Fig. 3). When the lineages of each sample determined using molecular phylogenetic analysis was reconstructed in the haplotype network, each sample was integrated in the same group as the lineages in the phylogenetic analysis (e.g., haplotype group A corresponds to lineage A in molecular phylogenetic analysis).

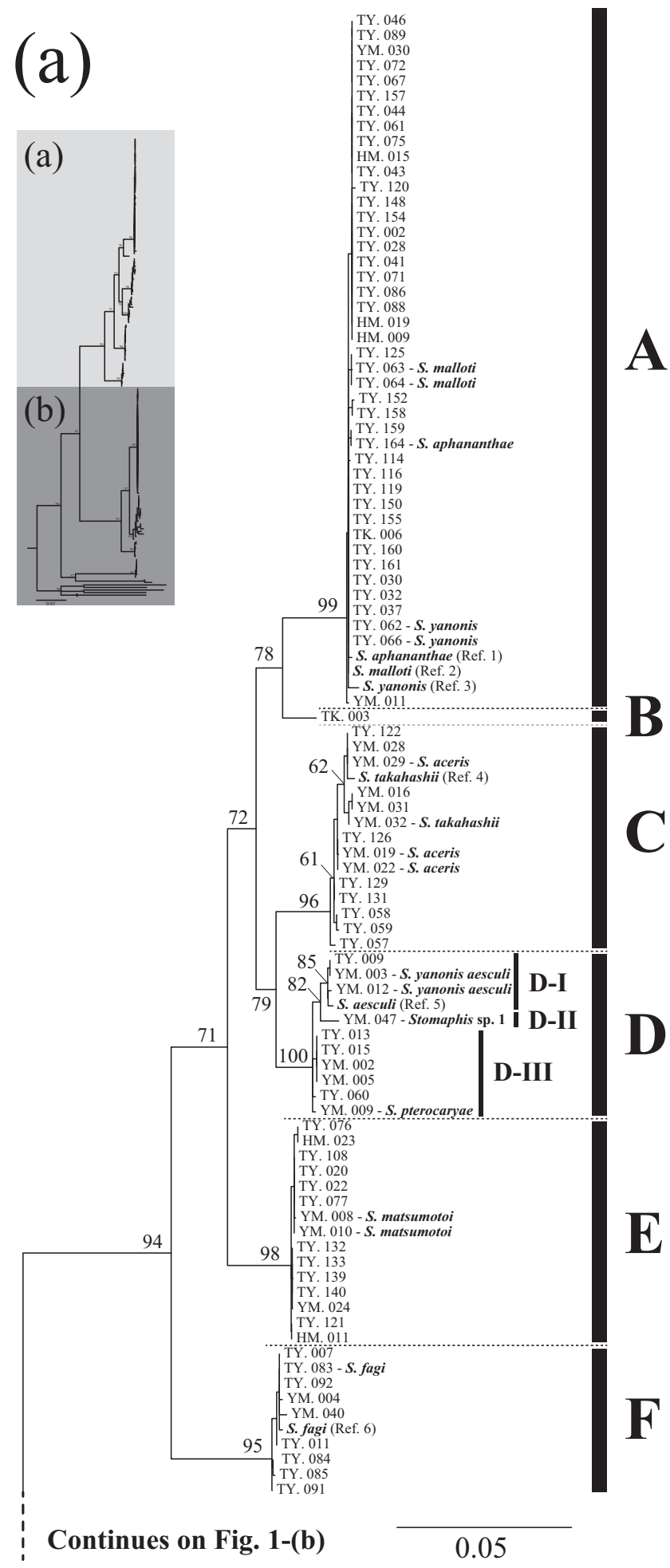

Fig. 1 Maximum likelihood phylogenetic tree of Stomaphis aphid samples based on mitochondrial COII and nuclear $E F-1 \alpha$ sequences. The inset at the top left shows an overview of the complete tree and the parts shown in $\mathbf{a}$ and $\mathbf{b}$. The tree shows ten major lineages (A to J). Lineages $\mathrm{D}$ and $\mathrm{H}$ each comprise several sublineages (D-1 to D-3, H-1 to H-5). The sample number and species, either morphologically identified or referenced from GenBank (if known), are shown for each operational taxonomic unit. See Table S1 for details of the samples. The bootstrap probability is shown for each node, and the scale indicates a nucleotide substitution rate of 0.05 


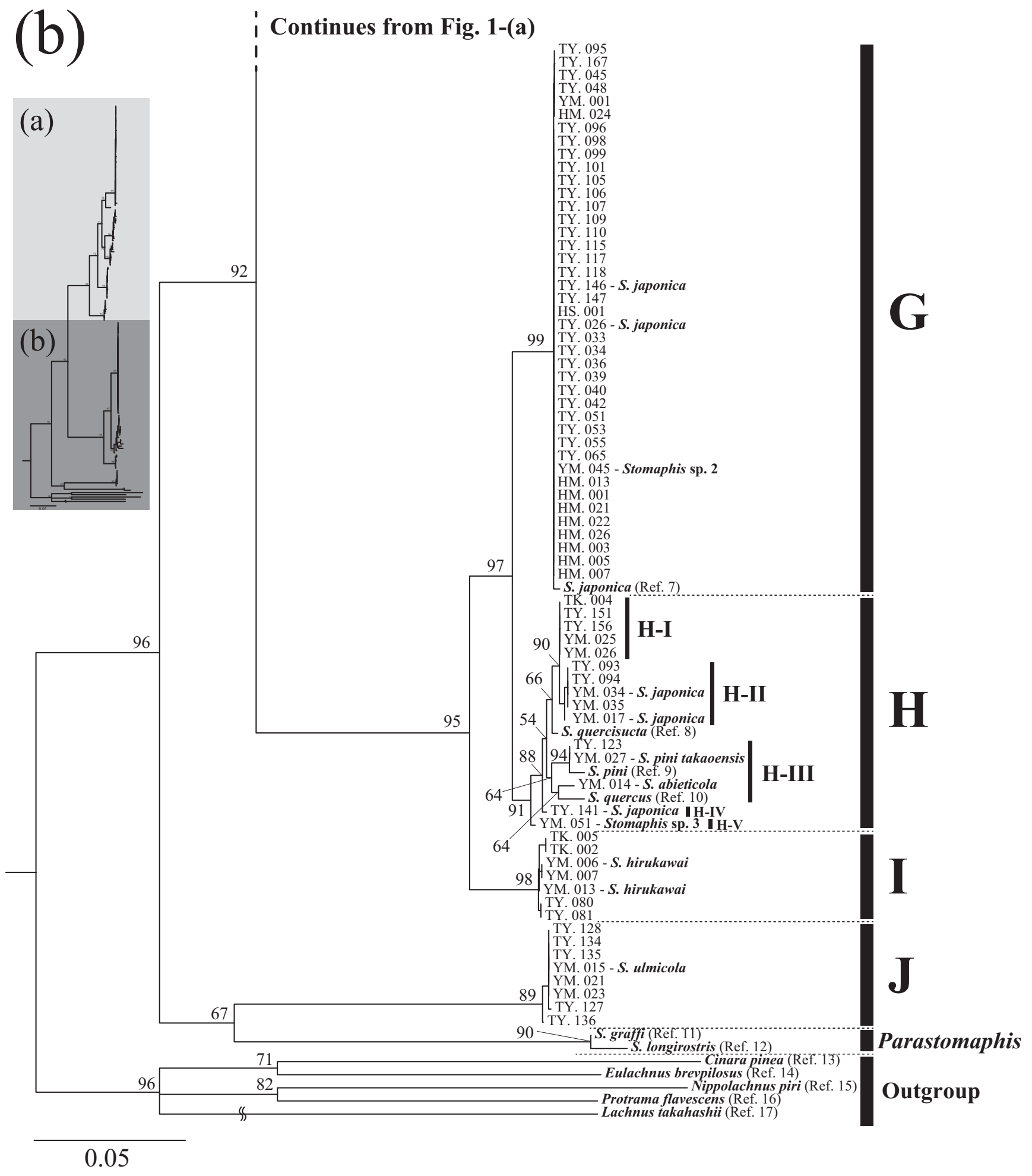

Fig. 1 (continued)

Some haplotypes were detected in haplotype groups C, E, $\mathrm{F}, \mathrm{I}$, and $\mathrm{J}$, and all haplotypes in these groups used single plant species. In haplotype group A, seven haplotypes were detected. The haplotypes A1, A4, and A5 used multiple plant species. Conversely, haplotypes A2, A3, A6, and A7 used single plant species, but these plant species overlapped with 
Fig. 2 Specificity of Stomaphis lineages to host plant and associated ant species. Letters in the left column indicate the DNA lineages inferred by molecular phylogenetic analysis by using mitochondrial and nuclear DNA sequences (Fig. 1). The pie charts show the compositions of host plant species and associated ant species and subgenera and genera for each aphid lineage (see the

Supporting information,

Tables S2 and S3, for the complete data set). For lineages D and $\mathrm{H}$, a host plant pie chart is also shown for each sublineage. The number of samples (number of aphid colonies for which the host plant or associated ant were identified) $N$ is shown below each pie chart

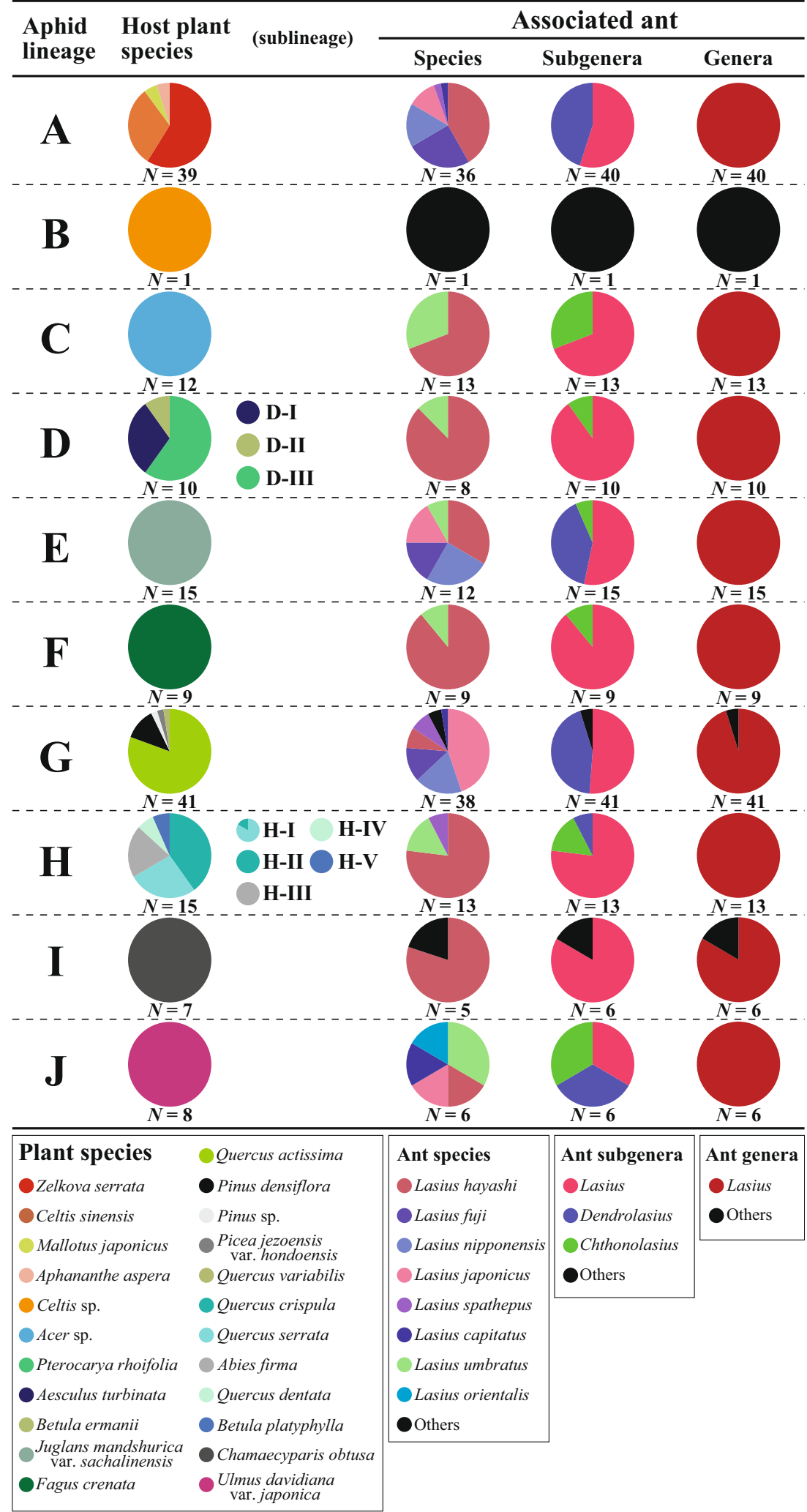

other haplotypes in group A. In haplotype group D, six haplotypes were detected. Each haplotype uses a single plant species. In haplotype group $\mathrm{G}$, one haplotype using multiple plant species was detected. In haplotype group $\mathrm{H}$, seven haplotypes were detected. Except for haplotype H5, each haplotype used a single plant species. Haplotype H5 used two plant species, 


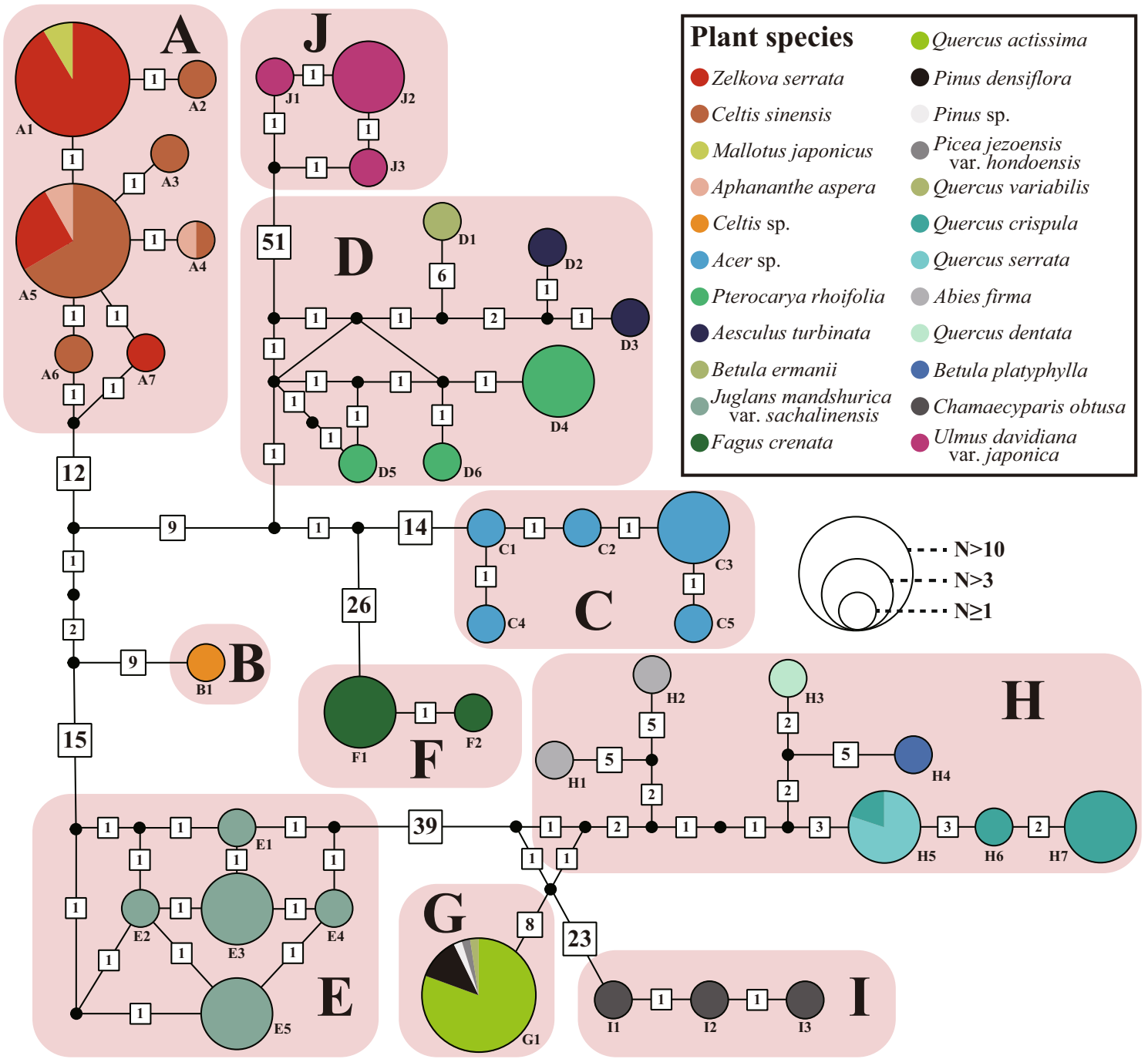

Fig. 3 Mitochondrial haplotype network of Stomaphis aphids. Numbers near the circle indicate haplotype numbers (see Table S1). The size of the circle indicates the number of samples of the haplotype. The color of the

one of which is the same species used by haplotypes H6 and H7.

\section{Mutualistic association with ants}

All of the investigated Stomaphis aphid colonies were attended by ant workers. Ants of genus Lasius were the most frequent (97\%; 150/154 aphid colonies; Tables S2 and S3). Considering their $C O I$ nucleotide sequences, eight Lasius ant species belonging to three subgenera (Lasius, Dendrolasius, and Chthonolasius) were identified. Other observed attending ant species were Camponotus obscuripes, Crematogaster sp., and Polyrhachis lamellidens (Table S1).

The ant subgenus Lasius accounted for 61\% (95/154 aphid colonies) of all associated ants, and it was the most frequent subgenus among ants associated with all Stomaphis aphid lineages except lineage $\mathrm{J}(N=6$; Fig. 2). The ant subgenus circle indicates the proportion of plants used by the haplotype. The number in the box indicates the number of mutations

Dendrolasius accounted for 29\% (45/154) of all associated ants, and ants of this subgenus attended aphids of five Stomaphis lineages (A, E, G, H, and J). The ant subgenus Chthonolasius accounted for 7\% (11/154) of all associated ants and attended aphids of six Stomaphis lineages (C, D, E, F, H, and J).

\section{Discussion}

Phylogenetic analysis of Stomaphis aphids and their relationships with host plants and attending ants revealed that each lineage and haplotype of Stomaphis aphids showed a high degree of specificity to host plant species, and no specieslevel host plant overlap was noted among lineages. Conversely, almost all lineages of Stomaphis aphids were associated with two or more ant species. These findings suggest that Stomaphis evolved and diversified owing to host plant 
shifts, whereas diversification rarely followed associated ant shifts. In addition, most Stomaphis aphid lineages were associated exclusively with ants of the genus Lasius, indicating that the mutualism between Stomaphis and Lasius has been very tight.

\section{Species-specific host plant use in Stomaphis}

Most of the DNA lineages and haplotypes of Stomaphis aphids used a single plant species as host (Figs. 2 and 3). In general, aphids have high specificity to their host plants because of the need to adapt and specialize to plant speciesspecific traits such as nutrient composition, defense systems (external morphology and secondary metabolites), and phenology (Dixon 1998; Peccoud et al. 2010). Stomaphis aphids can also benefit by adapting physiologically, morphologically, and ecologically to plant species-specific traits. Thus, like other phytophagous insects (War et al. 2012), Stomaphis aphids may exhibit a pattern of specificity for a particular plant species.

\section{Evolution of host plant use in Stomaphis}

Stomaphis aphids use a phylogenetically broad range of host plants. In Japan, their host plants belong to ten families in five orders (Table S2); globally, their host plants belong to 13 families in seven orders (Blackman and Eastop 2019). A conspicuous result of this study is that, in Japan, host plant shifts of Stomaphis aphids have occurred between taxa that are widely separated phylogenetically. Phylogenetic constraints on host plant utilization by aphids usually occur, and many aphid genera or families are associated strictly with a single plant genus or family (Peccoud et al. 2010). Because plant phenology, chemical compounds, and nutritional value are similar among closely related host plant species (Prasad et al. 2012; Davies et al. 2013), host shifts by aphids may occur only between plants belonging to, for example, a single genus. For example, conifer-feeding aphids of the genus Cinara, belonging to the same subfamily, Lachninae, as the genus Stomaphis, comprise as many as 250 species worldwide, all of which use host plants belonging to three families (Pinaceae, Cupressaceae, and Taxaceae) in the order Pinales (Blackman and Eastop 2019). Thus, the phylogenetic breadth of host plants used by the Stomaphis aphids in our study is clearly different from the general pattern of aphid diversification. Moreover, this host plant use pattern probably does not reflect the extinction of intermediate lineages, because relatively closely related aphid lineages, such as the sublineages in $\mathrm{D}$ and $\mathrm{H}$, show host shifts to distantly related plant taxa. For example, within lineage $\mathrm{D}$, the sublineages reflect host shifts from Pterocarya and Betula (Fagales) to Aesculus (Sapindales; Fig. 1 and Table S2).
Similar evolutionary host shifting by phloem sap feeders has been shown in the treehopper Enchenopa binotata species complex; each host lineage in this complex is specific to a single plant species, but together the host lineages use various plant taxa (Wood and Guttman 1983). Wood and Guttman (1983) inferred that this pattern reflects, first, fidelity to a single plant species, which arises because specialization on a particular plant species is advantageous for each host lineage; second, a release from phylogenetic constraints that enables the occurrence of shifts between distantly related plant species (Wood and Keese 1990; Wood 1993; Hsu et al. 2018).

As noted above, specializing physiologically, morphologically, and ecologically to a single host plant species is advantageous for Stomaphis aphids. Subsequently, they may release from phylogenetic constraints such as nutrient value, defense systems, and phenology of plant species. In lineage $\mathrm{H}$, host shift was noted between angiosperm (Abies) and gymnosperm (Quercus) trees. The nutrient value, secondary metabolite composition, and bark morphology differed between the two groups. For example, Abies tree has the resin composed of terpenes, which may be assumed to be considerably toxic for insects, and Stomaphis aphids must overcome this toxic resin when they use Abies tree as their host. Therefore, the acquisition of novel host plants for Stomaphis aphids may not be restricted strongly by plant physiological and morphological traits. In addition, because the tree trunk stores large amounts of nutrients throughout the season, Stomaphis aphids can use the phloem sap and survive even in winter (Depa 2013; Depa et al. 2015b). That is, seasonal variations of nutrients does not affect Stomaphis aphids; in contrast, almost all other aphid species need to ecologically adapt to seasonal variations of nutrients (e.g., host alternation, aestivation, and galling). Therefore, when Stomaphis aphids switch to novel host plants, they may not be threatened by the differences of plant phenology, thereby weakening the phylogenetic constraints for the availability of plant use.

\section{Does strong dependence on ant mutualism affect the pattern of plant use?}

Stomaphis aphids have a sedentary life mode, possibly because of their large body size, which is necessary for sucking phloem sap from tree trunks and consequent low dispersal ability. This sedentary life mode leads in turn to their strong dependence on ant mutualism (Depa et al. 2015a). Moreover, their sedentary life mode suggests that gene flow between aphids on different host plants would be extremely low, which would promote disruptive selection. As in the treehopper E. binotata species complex (Wood and Guttman 1983), a sedentary life mode owing to their dependence on ant mutualism may be one of the factors promoting disruptive selection in Homoptera by using different host plants (Wood 1982, 1987). Moreover, Depa et al. (2017) showed that more 
sedentary species of Stomaphis exhibit greater genetic variation and use a broader range of host plant taxa than less sedentary sister species found in the same area.

Depa et al. (2017) have shown that Stomaphis aphids can be accidentally transferred to neighboring tree species by attending ants. The results of this study also suggest that Stomaphis aphids might have dispersed in this manner. If transfer by attending ants occurs, then "generalist" Stomaphis species might use host plant species that, although distantly related taxonomically, have similar environmental preferences and are distributed sympatrically. Indeed, although the four host plant species used by Stomaphis lineage A belong to three different plant families, they all are found in a sunny, dry, and lowland forest edge environment. Similarly, the three host plant species used by Stomaphis lineage D belong to three different plant families, but grow in moist mountain forest environments. Determination of the factors that have led Stomaphis aphids to shift to distantly related host plants and their consequent evolutionary diversification is a topic for future studies. Such studies would allow us to gain insights into the mechanisms for the diversification of phytophagous insects.

\section{Evolution of mutualistic ant association in Stomaphis}

In this study, most Stomaphis colonies were associated with ants of the genus Lasius (Fig. 2), suggesting that mutualism with Lasius ants is important for the survival of Stomaphis aphids. Lasius ants usually nest at the base of trees (Terayama et al. 2014); hence, Stomaphis aphids living on tree trunks may be more likely to encounter Lasius ants than ants of other genera. In addition, Lasius worker ants walk up tree trunks in large numbers to collect food resources high up on the tree (Terayama et al. 2014); therefore, they can easily defend aphids living on the tree trunks against their natural enemies. Moreover, Lasius ant colonies persist for long periods of several years or more (Matsuura and Yashiro 2006), which enhances their ability to act as a stable partner of sedentary Stomaphis aphids. The strong defense provided to Stomaphis aphids by Lasius ants can compensate for the aphids' low escape ability owing to their large body and long proboscis. Future investigations of survival and reproduction rate differences between Stomaphis colonies associated with Lasius and those associated other ant taxa should provide further insight into the evolution of this aphid-ant mutualism.

A high proportion of aphid colonies in the Stomaphis lineages were attended by ants of the subgenus Lasius (Fig. 2), which are among the most common ants in Japan and occur in a wide range of environments from bare land to forest (Terayama et al. 2014). In addition, Matsuura and Yashiro (2006) reported that ants of the subgenus Lasius build shelters made of soil over Stomaphis aphid colonies on tree trunks and protect
Stomaphis aphid eggs in their nests during the winter. These ant behaviors suggest that ants of this subgenus are among the most useful mutualistic partners for Stomaphis.

Aphids of several lineages were attended by ants of the subgenus Dendrolasius in relatively low proportion. Establishment of a mutualistic association between Stomaphis aphids and ants of the subgenus Dendrolasius might be difficult. The morphological traits of the European aphid S. quercus (dark, slender, shiny body, and a strong degree of cuticle sclerotization) make them inconspicuous to natural enemies and tolerant of a harsh environment and also well adapted to Dendrolasius ant protection, because these ants do not build shelters over Stomaphis aphid colonies on tree trunks, but directly attend the aphids (Depa et al. 2017). In our survey, we found many Dendrolasius ant colonies in Tokamachi, Niigata Prefecture; however, although aphid colonies of lineage A (TY.089) were attended by Dendrolasius ants, those of lineage F observed in this area (TY.083, TY.084, TY.085, TY.091, and TY.092) were not. In addition, morphologically, S. yanonis (lineage A) are dark and slender, but S. fagi (lineage F) are white and round (Matsumoto 2008). These facts suggest that only species such as $S$. yanonis and $S$. quercus, which have acquired certain morphological traits, can associate with the ant subgenus Dendrolasius. In the future, investigation of the comparative morphology of many Stomaphis species in relation to their associated attending ant species would likely reveal the ant mutualism-related adaptations in Stomaphis.

Species specificity to the associated ant species has been reported in many obligate ant mutualisms (e.g., between ants and plants (Quek et al. 2004), or ants and Lycaenid butterflies (Pierce et al. 2002)). In Stomaphis aphids, Depa et al. (2017) reported one example where speciation was apparently driven by ant-aphid interactions. However, the Stomaphis lineages identified in this study were not associated with specific ant species. Endo and Itino (2012) showed that S. yanonis (lineage A in this study) successfully avoids attack and maintains its intimate relationship with its attending ant species, Lasius fuji, by having cuticular hydrocarbons similar to those of $L$. fuji worker ants. This finding suggests that $S$. yanonis is adapted to a specific attending ant species. However, colonies in lineage A were associated with not only $L$. fuji but also other ant species; one explanation may be that $S$. yanonis adapt locally to different ant species by changing their cuticular hydrocarbon, in areas where the density of $L$. fuji ants is low.

\section{Classification of Stomaphis}

Until recently, aphids of the genus Stomaphis were classified on the basis of their morphological characteristics, although, in some cases, the morphological classification of Stomaphis has been modified on the basis of 
phylogenetic relationships reconstructed using genetic markers (Depa and Mróz 2013). The molecular phylogeny of Stomaphis in Japan reconstructed in this study differed in part from the morphological classification, suggesting that the classification of some Japanese species, for example, of lineages A, D, G, and H, should be revised. However, no colonies of $S$. alni and $S$. carpini, other Stomaphis species that have been described in Japan (Sorin 1965), were sampled in this study. Therefore, more extensive sampling and more detailed morphological and ecological information are necessary to classify accurately Japanese Stomaphis.

In particular, Takada (2008) conducted fragmentary observations over 10 years and indicated that $S$. japonica may alternate hosts between Quercus serrata as primary and Quercus acutissima as secondary, although such host alternation is rare in Lachninae. In this study, the aphids using $Q$. serrata and $Q$. acutissima as host belonged to different lineages (lineages G and H). Stomaphis aphids on Q. acutissima certainly alternate their hosts because they fly away from $Q$. acutissima during winter. Therefore, Stomaphis aphids on $Q$. serrata and $Q$. acutissima might be different species, and aphids using $Q$. acutissima might have different primary host plants. Although a previous study was conducted in Kyoto (Takada 2008), we could not cover this area. Further investigation of the morphology and life history of lineages $G$ and $\mathrm{H}$ in a wide area is needed to reveal the host alternation in Stomaphis.

Acknowledgments We thank Takashi Komatsu, Hiroaki Miyairi, Hideaki Sekine, and Hiroyuki Yoshitomi for collecting Stomaphis specimens. We thank Susan T Duhon (Rujuke Editorial Service) and Editage (www.editage.com) for comprehensive English editing of this manuscript.

Funding information This work was supported by Grants-in-Aid for a Research Fellow from the Japan Society for the Promotion of Science: KAKENHI Grant No. 26291090 to T Itino and No. 16J09182 to T Yamamoto.

\section{Compliance with ethical standards}

Conflict of interest The authors declare that they have no competing interests.

Open Access This article is licensed under a Creative Commons Attribution 4.0 International License, which permits use, sharing, adaptation, distribution and reproduction in any medium or format, as long as you give appropriate credit to the original author(s) and the source, provide a link to the Creative Commons licence, and indicate if changes were made. The images or other third party material in this article are included in the article's Creative Commons licence, unless indicated otherwise in a credit line to the material. If material is not included in the article's Creative Commons licence and your intended use is not permitted by statutory regulation or exceeds the permitted use, you will need to obtain permission directly from the copyright holder. To view a copy of this licence, visit http://creativecommons.org/licenses/by/4.0/.

\section{References}

Agosta SJ (2006) On ecological fitting, plant-insect associations, herbivore host shifts, and host plant selection. Oikos 114:556-565. https://doi.org/10.1111/j.2006.0030-1299.15025.x

Blackman RL, Eastop VF (1994) Aphids on the world's trees: an identification and information guide. CAB International, Wallingford

Blackman RL, Eastop VF (2019) Aphids on the world's plants: an online identification and information guide. http://www. aphidsonworldsplants.info. Accessed 10 April 2019

Brower AV, Jeansonne MM (2004) Geographical populations and "subspecies" of new world monarch butterflies (Nymphalidae) share a recent origin and are not phylogenetically distinct. Ann Entomol Soc Am 97:519-523. https://doi.org/10.1603/0013-8746(2004) 097[0519:GPASON]2.0.CO;2

Brożek J, Mróz E, Wylężek D, Depa Ł, Węgierek P (2015) The structure of extremely long mouthparts in the aphid genus Stomaphis Walker (Hemiptera: Sternorrhyncha: Aphididae). Zoomorphology 134: 431-445. https://doi.org/10.1007/s00435-015-0266-7

Davies TJ, Wolkovich EM, Kraft NJB et al (2013) Phylogenetic conservatism in plant phenology. J Ecol 101:1520-1530. https://doi.org/ $10.1111 / 1365-2745.12154$

Depa $\_$(2013) Life cycle of maple-tree aphid Stomaphis graffii Cholodkovsky, 1894 (Hemiptera, Aphididae). Anim Biol 63:313320. https://doi.org/10.1163/15707563-00002414

Depa Ł, Mróz E (2013) Central European Acer- and Salicaceae-feeding aphids of the genus Stomaphis (Insecta: Aphidoidea: Lachnidae) separate species or populations? Zool Sci 30:509-518. https://doi. org/10.2108/zsj.30.509

Depa Ł, Mróz E, Szawaryn K (2012) Molecular identity of Stomaphis quercus (Hemiptera: Aphidoidea: Lachnidae) and description of a new species. Eur J Entomol 109:435-444. https://doi.org/10.14411/ eje.2012.056

Depa $€$, Kanturski M, Junkiert $Ł$, Wieczorek K (2015a) Giant females vs dwarfish males of the genus Stomaphis Walker (Hemiptera: Aphididae) - an aphid example of the ongoing course to permanent parthenogenesis. Arthropod Syst Phylo 73:19-40

Depa L, Taszakowski A, Kanturski M (2015b) Impact of warm weather events on prolongation of the life cycle of Stomaphis Walker (Hemiptera, Aphididae, Lachninae). Turk J Zool 39:669-671. https://doi.org/10.3906/zoo-1404-6

Depa $€$, Mróz E, Bugaj-Nawrocka A, Orczewska A (2017) Do ants drive speciation in aphids? A possible case of ant-driven speciation in the aphid genus Stomaphis Walker (Aphidoidea, Lachninae). Zool J Linnean Soc 179:41-61. https://doi.org/10.1111/zoj.12437

Dixon AFG (1998) Aphid ecology, 2nd edn. Chapman \& Hall, London

Drès M, Mallet J (2002) Host races in plant-feeding insects and their importance in sympatric speciation. Philos Trans R Soc Lond Ser B Biol Sci 357:471-492. https://doi.org/10.1098/rstb.2002.1059

Endo S, Itino T (2012) The aphid-tending ant Lasius fuji exhibits reduced aggression toward aphids marked with ant cuticular hydrocarbons. Popul Ecol 54:405-410. https://doi.org/10.1007/s10144-012-0314-9

Fischer MK, Shingleton AW (2001) Host plant and ants influence the honeydew sugar composition of aphids. Funct Ecol 15:544-550. https://doi.org/10.1046/j.0269-8463.2001.00550.x

Fujiyama N, Ueno H, Kahono S, Hartini S, Matsubayashi KW, Kobayashi N, Katakura H (2013) Distribution and differentiation of Henosepilachna diekei (Coleoptera: Coccinellidae) on two hostplant species across Java, Indonesia. Ann Entomol Soc Am 106: 741-752. https://doi.org/10.1603/an13053

Funk DJ, Futuyma DJ, Orti G, Meyer A (1995) A history of host associations and evolutionary diversification for Ophraella (Coleoptera: Chrysomelidae): new evidence from mitochondrial DNA. Evolution 49:1008-1017. https://doi.org/10.1111/j.1558-5646.1995.tb02335.x 
Futuyma DJ, Agrawal AA (2009) Macroevolution and the biological diversity of plants and herbivores. Proc Natl Acad Sci U S A 106: 18054-18061. https://doi.org/10.1073/pnas.0904106106

Grimaldi D, Engel MS (2005) Evolution of the insects. Cambridge University Press, Cambridge

Heie OE (1987) Morphological structures and adaptations. In: Minks AK, Harrewijn P (eds) Aphids: their biology, natural enemies, and control. Elsevier, Amsterdam, pp 393-400

Hölldobler B, Wilson EO (1990) The ants. Harvard University Press, Cambridge

Hsu YH, Cocroft RB, Snyder RL, Lin CP (2018) You stay, but I hop: host shifting near and far co-dominated the evolution of Enchenopa treehoppers. Ecol Evol 8:1954-1965. https://doi.org/10.1002/ece3.3815

Inouye M (1938) On three aphids of Lachninae from Hokkaido and Saghalien. Insecta matsumurana 12:74-80

Jobb G, Von Haeseler A, Strimmer K (2004) TREEFINDER: a powerful graphical analysis environment for molecular phylogenetics. BMC Evol Biol 4:18. https://doi.org/10.1186/1471-2148-4-18

Kanturski M, Karcz J, Kaszyca N, Depa $Ł$ (2017) Perianal structures in myrmecophilous subterranean aphids (Insecta: Hemiptera: Aphididae) - comparative morphology of trophobiotic organ with its first description in Lachninae. Arthropod Struct Dev 46:496507. https://doi.org/10.1016/j.asd.2017.06.001

Kozarzhevskaya EF (1986) Scale insects (Homoptera, Coccoidea) of ornamental plants in the European part of the USSR and some neighboring countries. Entomol Rev 64:144-158

Kumar S, Stecher G, Tamura K (2016) MEGA7: Molecular Evolutionary Genetics Analysis version 7.0 for bigger datasets. Mol Biol Evol 33: 1870-1874. https://doi.org/10.1093/molbev/msw054

Lang C, Menzel F (2011) Lasius niger ants discriminate aphids based on their cuticular hydrocarbons. Anim Behav 82:1245-1254. https:// doi.org/10.1016/j.anbehav.2011.08.020

Leigh JW, Bryant D (2015) Popart: full-feature software for haplotype network construction. Methods Ecol Evol 6:1110-1116. https://doi. org/10.1111/2041-210X.12410

Lorenz H, Scheurer S (1998) Biology and generation-order of Stomaphis quercus (Lachnidae) living on Betula pendula near Berlin, Germany. In: Nieto Nafría JM, Dixon AFG (eds) Aphids in natural and managed ecosystems. Universidad de León, León, pp 243-250

Maruyama M, Steiner FM, Stauffer C, Akino T, Crozier RH, SchlickSteiner BC (2008) A DNA and morphology based phylogenetic framework of the ant genus Lasius with hypotheses for the evolution of social parasitism and fungiculture. BMC Evol Biol 8:237. https:// doi.org/10.1186/1471-2148-8-237

Matsubayashi KW, Kahono S, Katakura H (2011) Divergent host plant specialization as the critical driving force in speciation between populations of a phytophagous ladybird beetle. J Evol Biol 24:14211432. https://doi.org/10.1111/j.1420-9101.2011.02274.x

Matsubayashi KW, Kahono S, Hartini S, Katakura H (2013) Microspatial and seasonal distributions of two sympatric host races of the phytophagous ladybird beetle Henosepilachna diekei (Coleoptera: Coccinellidae) and their host plants in West Java, Indonesia. Treubia 40:9-24. https://doi.org/10.14203/treubia.v40i0. 181

Matsumoto Y (2008) A guide illustrated book of aphids. Zenkokunousonkyouikukyoukai, Tokyo

Matsuura K, Yashiro T (2006) Aphid egg protection by ants: a novel aspect of the mutualism between the tree-feeding aphid Stomaphis hirukawai and its attendant ant Lasius productus. Naturwissenschaften 93:506-510. https://doi.org/10.1007/s00114006-0136-8

Normark BB (1999) Evolution in a putatively ancient asexual aphid lineage: recombination and rapid karyotype change. Evolution 53: 1458-1469. https://doi.org/10.1111/j.1558-5646.1999.tb05410.x
Nosil P, Crespi BJ, Sandoval CP (2002) Host-plant adaptation drives the parallel evolution of reproductive isolation. Nature 417:440-443. https://doi.org/10.1038/417440a

Novgorodova TA (2005) Ant-aphid interactions in multispecies ant communities: some ecological and ethological aspects. Eur J Entomol 102:495-501. https://doi.org/10.14411/eje.2005.071

Peccoud J, Ollivier A, Plantegenest M, Simon JC (2009) A continuum of genetic divergence from sympatric host races to species in the pea aphid complex. Proc Natl Acad Sci U S A 106:7495-7500. https:// doi.org/10.1073/pnas.0811117106

Peccoud J, Simon JC, von Dohlen C, Coeur d'acier A, Plantegenest M, Vanlerberghe-Masutti F, Jousselin E (2010) Evolutionary history of aphid-plant associations and their role in aphid diversification. C R Biol 333:474-487. https://doi.org/10.1016/j.crvi.2010.03.004

Pierce NE, Braby MF, Heath A, Lohman DJ, Mathew J, Rand DB, Travassos MA (2002) The ecology and evolution of ant association in the Lycaenidae (Lepidoptera). Annu Rev Entomol 47:733-771. https://doi.org/10.1146/annurev.ento.47.091201.145257

Prasad KVSK, Song BH, Manning CO et al (2012) A gain-of-function polymorphism controlling complex traits and fitness in nature. Science 337:1081-1084. https://doi.org/10.1126/science.1221636

Quek SP, Davies SJ, Itino T, Pierce NE (2004) Codiversification in an antplant mutualism: stem texture and the evolution of host use in Crematogaster (Formicidae: Myrmicinae) inhabitants of Macaranga (Euphorbiaceae). Evolution 58:554-570. https://doi. org/10.1111/j.0014-3820.2004.tb01678.x

Shingleton AW, Stern DL, Foster WA (2005) The origin of a mutualism: a morphological trait promoting the evolution of ant-aphid mutualisms. Evolution 59:921-926. https://doi.org/10.1111/j.0014-3820. 2005.tb01766.x

Sorin M (1965) Three new species of the genus Stomaphis in Japan, with a redescription of $S$. yanonis Takahashi (Aphididae, Homoptera). Bull Osaka Prefect Univ Ser B 16:81-88

Sorin M (1979) Two new species of the genus Stomaphis (Aphididae, Homoptera) from Japan. Bull Kogakkan Univ 17:1-13

Sorin M (1995) Two new species and hitherto unknown sexual morphs of the genus Stomaphis Walker (Homoptera, Aphididae) from Japan. Bull Kogakkan Univ 33:145-163

Sorin M (2012) Two new species and 2 new subspecies of the genus Stomaphis Walker (Hemiptera, Aphididae) from Japan. Bull Kogakkan Univ 50:1-32

Stadler B, Dixon AF (2005) Ecology and evolution of aphid-ant interactions. Annu Rev Ecol Evol Syst 36:345-372. https://doi.org/10. 1146/annurev.ecolsys.36.091704.175531

Stern DL (1994) A phylogenetic analysis of soldier evolution in the aphid family Hormaphididae. Proc R Soc Lond B Biol Sci 256:203-209. https://doi.org/10.1098/rspb.1994.0071

Takada H (2008) Life cycles of three Stomaphis species (Homoptera: Aphididae) observed in Kyoto, Japan: possible host alternation of S. japonica. Entomol Sci 11:341-348. https://doi.org/10.1111/j. 1479-8298.2008.00276.x

Takahashi R (1960) Stomaphis of Japan (Aphididae, Homoptera). Bull Osaka Prefect Univ Ser B 10:1-8

Tanabe AS (2007) Kakusan: a computer program to automate the selection of a nucleotide substitution model and the configuration of a mixed model on multilocus data. Mol Ecol Notes 7:962-964. https://doi.org/10.1111/j.1471-8286.2007.01807.x

Terayama M, Kubota S, Eguchi K (2014) Encyclopedia of Japanese ants. Asakura Shoten, Tokyo

War AR, Paulraj MG, Ahmad T, Buhroo AA, Hussain B, Ignacimuthu S, Sharma HC (2012) Mechanisms of plant defense against insect herbivores. Plant Signal Behav 7:1306-1320. https://doi.org/10.4161/ psb. 21663

Wood TK (1982) Ant-attended nymphal aggregations in the Enchenopa binotata complex (Homoptera: Membracidae). Ann Entomol Soc Am 75:649-653. https://doi.org/10.1093/aesa/75.6.649 
Wood TK (1987) Host plant shifts and speciation in the Enchenopa binotata Say complex. In: Wilson MR, Nault LR (eds) Proceedings of 2nd international workshop on leafhoppers and planthoppers of economic importance. $\mathrm{CAB}$ International Institute of Entomology, London

Wood TK (1993) Speciation of the Enchenopa binotata complex (Insecta: Homoptera: Membracidae). In: Lees DR, Edwards D (eds) Evolutionary patterns and processes. UK Academic Press, London, pp 299-317

Wood TK, Guttman SI (1983) Enchenopa binotata complex: sympatric speciation? Science 220:310-312. https://doi.org/10.1126/science. 220.4594 .310
Wood TK, Keese MC (1990) Host-plant-induced assortative mating in Enchenopa treehoppers. Evolution 44:619. https://doi.org/10.2307/ 2409439

Yao I (2012) Ant attendance reduces flight muscle and wing size in the aphid Tuberculatus quercicola. Biol Lett 8:624-627. https://doi.org/ $10.1098 / \mathrm{rsbl} .2012 .0014$

Publisher's note Springer Nature remains neutral with regard to jurisdictional claims in published maps and institutional affiliations. 